implemented this approach by performing local selective area growth. First, they masked the titanium-coated glass substrate with $\mathrm{SiO}_{2}$ and then formed submicrometrediameter apertures by conventional lithography and dry etching processes. The high-temperature growth of GaN on the surface successfully produced micrometrescale defect-free pyramids.

The researchers deposited InGaN/ GaN quantum wells on the pyramid array to form the active region of the device. They then processed the device with a thin p-type doped GaN layer and an indium tin oxide layer to form the top electrode. Most of the micro-LEDs exhibited electroluminescence, although their colour and intensity varied across the wafer. The structural quality of these micro-LEDs was evident from the high internal quantum efficiency (52\%) deduced from photoluminescence measurements.
However, the on-wafer external quantum efficiency was quite low $(<1 \%)$, especially considering that pyramidal LEDs inherently allow for efficient light extraction without introducing any complex and costly processing steps.

The main limitation of this performance improvement seems to originate from the electrical injection side. The researchers measured strong leakage currents and higher driving voltages than state-of-theart LED technology. They attribute these non-ideal current-voltage characteristics to processing issues and non-optimal p-type GaN. It is likely that the challenge of maintaining a high p-type doping level on the pyramid facets is due to the higher incorporation of oxygen on these surface planes than on the conventional $c$-plane. The vicinity of the $\mathrm{SiO}_{2}$ mask might also be a severe performance issue. Nevertheless, these 3D micro-LEDs exhibited a luminance of $600 \mathrm{~cd} \mathrm{~m}^{-2}$, which is very promising for most applications. Ultimately, this work perfectly illustrates the advantages that result from embracing a change of paradigm in optoelectronic research, where devices are not made from perfect 2D layers but instead from an ensemble of 3D microor nanostructures.

Nicolas Grandjean and Raphaël Butté are at the Institute of Condensed Matter Physics, Ecole polytechnique fédérale de Lausanne (EPFL), Station 3, CH-1015, Lausanne, Switzerland. e-mail:nicolas.grandjean@epfl.ch

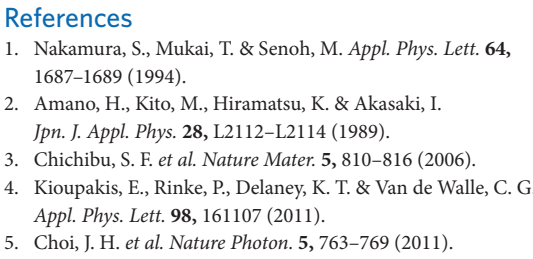

\title{
SOLID-STATE LASERS
}

\section{The Airy beam laser}

The Airy beam has attracted significant interest from the optics community, thanks to its peculiar curved propagating trajectory and non-diffracting and self-healing features. In recent years, many researchers have investigated the physics of this unique beam and explored its wealth of potential applications, which range from improving microscopy and the optical manipulation of particles to nonlinear frequency conversion and the generation of light bullets.

Airy beams are usually generated by passing a Gaussian beam emitted from a conventional laser through a phase mask, which imparts a cubic phase modulation across the beam. Now, having developed a method for realizing laser beams with arbitrary beam profiles, Gil Porat and coworkers from Tel Aviv University in Israel have built a solid-state laser whose emitted beam has a two-dimensional Airy intensity profile (Opt. Lett. 36, 4119-4121; 2011).

The key to the development is the use of an aperiodic binary reflection diffraction grating as the output mirror of a laser cavity. The grating reflects most of the light back into the cavity but allows some to leave via various diffraction orders. The diffraction grating therefore serves the role of the phase mask normally used for Airy beam generation, except that it is now an integral part of the laser.

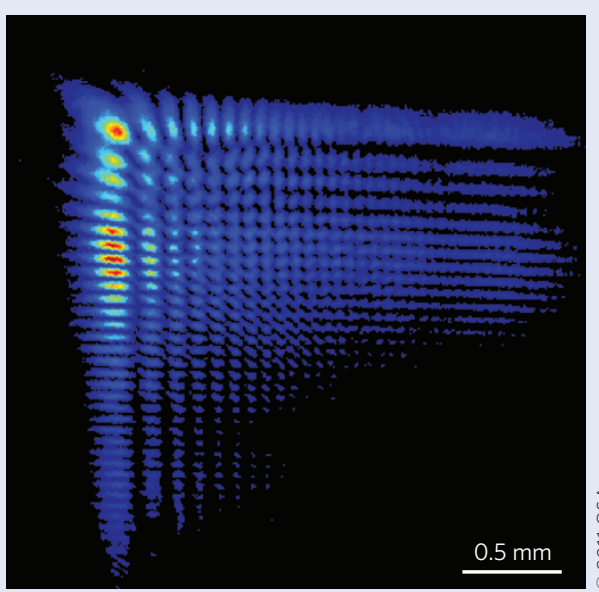

a total cavity length of $76 \mathrm{~mm}$. They then passed one of the first-order diffraction beams from the grating through a lens to form the optical Fourier transformation of the beam at the plane of the diffraction grating. The lasing threshold pump power was $1.4 \mathrm{~W}$ and the slope efficiency was $13.5 \%$, which corresponds to a maximum output power of $67.5 \mathrm{~mW}$ at a pump power of $1.94 \mathrm{~W}$. To verify that the output beam from the laser was indeed an Airy beam, the team successfully performed a simple self-healing experiment in which a knife was used to block the main lobe of the beam.

The researchers say that their approach is more compact and

The researchers fabricated a customdesigned silver-coated silicon grating using electron-beam writing to impose a transverse cubic phase, which is required to generate the Airy beam, on the diffracted light leaving the laser. The 70-nm-thick silver layer featured modulation patterns with a ridge height of $61 \mathrm{~nm}$ and provided a zero-order reflectivity of $87.6 \%$ and diffraction efficiency of $5 \%$ for each of the two first-order diffraction beams at a wavelength of 1,064 $\mathrm{nm}$.

The researchers placed the grating in an optical cavity formed by an $808 \mathrm{~nm}$ diode-pumped Nd:YAG laser system, giving convenient than the conventional method of generating Airy beams, which requires a phase-modulating element to be present outside the laser cavity. They also point out that the Airy beam laser is a proof-of-principle demonstration of a more general method currently under development that should also make it possible to build lasers and optical parametric oscillators capable of producing vortex beams and Bessel beams, or even beams with dynamically controllable and arbitrary profiles.

RACHEL WON 